\title{
THEOREM OF STERNBERG-CHEN MODULO CENTRAL MANIFOLD FOR BANACH SPACES
}

\author{
VICTORIA RAYSKIN
}

\begin{abstract}
We consider $C^{\infty}$-diffeomorphisms on a Banach space with a fixed point 0 . Suppose that these diffeomorphisms have $C^{\infty}$ non-contracting and non-expanding invariant manifolds, and formally conjugate along their intersection (the center). We prove that they admit local $C^{\infty}$ conjugation. In particular, subject to non-resonance condition, there exists a local $C^{\infty}$ linearization of the diffeomorphisms. It also follows that a family of germs with a hyperbolic linear part admits a $C^{\infty}$ linearization, which has $C^{\infty}$ dependence on the parameter of the linearizing family. The results are proved under the assumption that the Banach space allows a special extension of the maps. We discuss corresponding properties of Banach spaces. The proofs of this paper are based on the technique, developed in the works of G. Belitskii ([B1], [B2]).
\end{abstract}

\section{INTRODUCTION}

Approximations by normal forms and especially linear approximation are convenient simplifications of a physical system. Most of the physical systems are assumed to be analytic. The study of analytic conjugation was initiated by Poincaré and extended in numerous works. But it was shown in the work of Bruno $([\mathrm{Br}])$ that the class of systems, which admits analytic reduction to the Poincaré-Dulac normal form is small even in $\mathbb{R}^{n}$. For that reason we study this problem in the class of $C^{\infty}$ transformations.

This question was studied in numerous works (for example, in $[\mathrm{S}],[\mathrm{Ch}],[\mathrm{B}]$ ). The first step in the proofs of these results in $\mathbb{R}^{n}$ is the condition of formal solvability in $C^{\infty}$. The latter generates an algebraic expression, called resonance condition. For a generic diffeomorphism, the non-resonance assumption is a necessary condition for removing non-linear terms.

Although, resonance is an obstacle for a smooth conjugation in dimensions higher than two, for diffeomorphisms on $\mathbb{R}^{n}$, whose hyperbolic linear part, consists of only contraction (or only expansion) assumption of resonance can be removed (see $[\mathrm{H}]$ or [B4] for a more general result). The situation is qualitatively different in Banach spaces. It was shown in the work of [RS-M] that the non-resonance assumption in Banach space is essential even for contractions. They construct an example of a contraction diffeomorphism in infinite dimensions that is not $C^{1}$ linearizable.

In this paper we study the question of smooth conjugation of diffeomorphisms with linear part $\Lambda$. The diffeomorphisms are defined on a Banach space $B$. We

The author thanks G. R. Belitskii for many inspiring and valuable discussions during the preparation of this paper. 
assume that they have invariant smooth non-contracting and non-expanding manifolds $W_{U, 0}$ and $W_{S, 0}$, such that their intersection $W_{U, 0} \cap W_{S, 0}=W_{0}$ is the central manifold. If $\Lambda$ is hyperbolic, then $W_{U, 0} \cap W_{S, 0}=\{0\}$.

It is also interesting to consider the situation, when $W_{U, 0} \cap W_{S, 0}$ is a set of parameters of the family of diffeomorphisms with hyperbolic linear part. In this case, $B=B_{1} \times B_{2}$ and

$$
F(x, t)=\left\{\begin{array}{l}
\Lambda x+f(x, t) \\
t
\end{array}\right.
$$

for $x \in B_{1}$ and $t \in B_{2}$.

If we restrict our attention to the question of smooth dependence of linearizing homeomorphisms on the parameter, we do not need to make a non-resonance assumption for some class of such diffeomorphisms. It was shown in $[\mathrm{R}]$ that for a family of diffeomorphisms with hyperbolic linear part and with non-linearities of the second order, there exists a family of linearizing homeomorphisms which have $C^{1}$ dependence on the parameter. Unfortunately, the methods, developed in the paper can only be used for diffeomorphisms with resonances. Also, we only proved $C^{1}$ differentiability. The proof for higher regularity would be very routine.

In this paper we show that, subject to formal conjugation along the central manifold, diffeomorphisms admit a $C^{\infty}$ local conjugation. As a consequence, we deduce that subject to the non-resonance condition, there exists a $C^{\infty}$ local linearization of the family.

One of the technical difficulties is the question of $C^{\infty}$ extension of a local map to the whole space $B$. It is connected to the question of existence of "hat" functions, i.e., $\tau(y) \in C^{\infty}$ with bounded support. (One can also see the assumption about "hat" function in the proof of $C^{1}$ conjugation for contracting diffeomorphisms on Banach spaces in the paper of RS-M1.) If $\tau(y)$ is such a function, then the extension of $f$, call it $\tilde{f}$ can be defined as $\tilde{f}(y)=f(\tau(y) \cdot y)$. In this expression $\tau(y) \cdot y$ defines a map $K(y): B \rightarrow B(K(y)=\tau(y) \cdot y)$ with the following properties:

1. $K(y)=y$ for $\|y\|<1$

2. $K \in C^{\infty}$ and $\left\|K^{(j)}(y)\right\| \leq c_{j}, j=0,1, \ldots$

Therefore, we can see that for existence of extension $\tilde{f}$ it is enough to have map $K$, defined on the Banach space.

It turns out that there exist Banach spaces (for example, $C([0,1])$ ) without "hat" functions, but with $K$ maps. Moreover, the question of extension of a map remains open. It is not known whether there are Banach spaces without $K$ maps.

The methods, used in this paper, were developed in the two works of G. Belitskii: [B1], [B2]. The technique of the first article allows us to consider the problem in Banach spaces, where we use a special metric, induced by a countable collection of norms. The special metric (with "inner" contraction) permits us to use the Banach Contraction Principle on the bounded set of smooth maps. The idea to use the Banach Contraction Principle for the study of diffeomorphisms with nonhyperbolic linear part comes from the second paper, [B2], where the families of germs are studied.

\section{THE MAIN THEOREM}

Lets consider a linear operator $\Lambda$ on a Banach space $B$. It divides $B$ into contracting, central and expanding subspaces $L_{-}, L_{0}$ and $L_{+}$(see, for example [N]). 
Denote by $L_{-, 0}$ and $L_{+, 0}$ the linear subspaces of $B$ such that $\left|\sigma\left(\Lambda_{\mid L_{-, 0}}\right)\right| \leq 1$ and $\left|\sigma\left(\Lambda_{\mid L_{+, 0}}\right)\right| \geq 1$. (Here, $\sigma\left(\Lambda_{\mid L_{ \pm, 0}}\right)$ is the spectrum of the restriction of the operator $\Lambda$ on $L_{ \pm, 0}$.) Assume that there exist invariant non-contracting and nonexpanding manifolds $W_{U, 0}$ and $W_{S, 0}$ of $F$ which are $C^{\infty}$ graphs over $L_{+, 0}$ and $L_{-, 0}$ correspondingly. Denote by $W_{0}$ the intersection of $W_{U, 0}$ and $W_{S, 0}$.

Since $W_{U, 0}$ and $W_{S, 0}$ are $C^{\infty}$ graphs over $L_{+, 0}$ and $L_{-, 0}$, we can smoothly straighten $W_{U, 0}$ and $W_{S, 0}$. I. e., we shell assume that $W_{U, 0}=L_{+, 0}$ and $W_{S, 0}=L_{-, 0}$. Also, lets write $B=B_{1} \times B_{2}$, where $B_{1}=L_{-} \oplus L_{+}$and $B_{2}=L_{0}$.

Lets assume that there exist $K_{+}, K_{0}$ and $K_{-}$, defined on $L_{+}, L_{0}$ and $L_{-}$correspondingly, with the properties $1-2$, discussed above. Then, it is clear that the map $K: B \rightarrow B$ can be defined as $K=\left(K_{+}, K_{0}, K_{-}\right)$. But it is unknown, whether the existence of $K$ on $B$ implies the existence of $K_{ \pm}, K_{0}$ on the subspaces. Thus, assuming that there exist maps $K_{ \pm}, K_{0}$ we can prove the following theorem.

Theorem 1. Assume that there exist $C^{\infty}$ invariant non-expanding and non-contracting manifolds, and there exist maps $K_{ \pm}$and $K_{0}$ on $L_{ \pm} \subset B_{1}$ and on $L_{0} \subset B_{2}$. Let

$$
F(y)=\Lambda y+f(y)
$$

and

$$
G(y)=\Lambda y+g(y)
$$

be two $C^{\infty}(B)$ diffeomorphisms. Assume that $f(y)=o(\|y\|), g(y)=o(\|y\|)$ and $f(y)-g(y)=o\left(\left(\operatorname{dist}\left(y, L_{0}\right)\right)^{n}\right)$ for all $n \in \mathbb{N}$, as $\operatorname{dist}\left(y, L_{0}\right) \rightarrow 0$.

Then, there exists a $C^{\infty}(B)$ diffeomorphism $H(y)=y+h(y)$, with $h(y)=$ $o(\|y\|)$, as $\|y\| \rightarrow 0$ and $h(y)=o\left(\left(\operatorname{dist}\left(y, L_{0}\right)\right)^{n}\right)$ for all $n \in \mathbb{N}$, as $\operatorname{dist}\left(y, L_{0}\right) \rightarrow 0$, such that for small values of $\|y\|$

$$
H \circ F=G \circ H .
$$

Proof. We are interested in the local properties of the maps $F$ and $G$. Thus, we can modify the maps $F$ and $G$ with the help of $K$, so that the new maps coincide with the original maps in a small $\delta$ neighborhood of the origin. From now on we shell assume that

$$
F(y)=\Lambda y+f(\delta \cdot K(y / \delta))
$$

and

$$
G(y)=\Lambda y+g(\delta \cdot K(y / \delta))
$$

The first derivatives of the non-linear parts of these maps are close to 0 and all other derivatives are bounded. Thus, we obtained the desired extensions of these maps to $B$.

It is enough to prove the existence of a $C^{\infty}$ solution $h$ for the equation

$$
(\mathrm{Id}+h) \circ(\Lambda+f)=(\Lambda+g) \circ(\mathrm{Id}+h) .
$$

Consider closed subspaces of $C^{\infty}$ maps:

$$
\mathcal{E}_{L_{+, 0}}=\left\{\phi \in C^{\infty}(B): \phi_{y \in L_{+, 0}}^{(k)}=0, k \in \mathbb{N}\right\}
$$

and

$$
\mathcal{E}_{L_{-, 0}}=\left\{\phi \in C^{\infty}(B): \phi_{y \in L_{-, 0}}^{(k)}=0, k \in \mathbb{N}\right\}
$$

We shell say that $\phi$ is flat on $L_{-, 0}$ (or $L_{+, 0}$ ), if $\phi \in \mathcal{E}_{L_{-, 0}}$ (or $\phi \in \mathcal{E}_{L_{+, 0}}$ ). The equation (4) can be reduced to

$$
h(\Lambda y+f(y))-\Lambda h(y)-(g(y+h(y))-g(y))=g(y)-f(y)
$$


The right side of this equation (call it $\gamma$ ) is flat on $L_{0}$. By Lemma $10, \gamma$ can be written as a sum of two maps, $\gamma_{+}$and $\gamma_{-}$, flat on $L_{-, 0}$ and $L_{+, 0}$ correspondingly. We shell search for a solution $h$ of equation 5 as a sum of two solutions, $h_{+} \in \mathcal{E}_{L_{+, 0}}$ and $h_{-} \in \mathcal{E}_{L_{-, 0}}$, where $h_{+}$satisfies

$$
\begin{aligned}
h_{+}(y) & =\Lambda h_{+}\left((\Lambda y+f(y))^{-1}\right) \\
& +g\left((\Lambda y+f(y))^{-1}+h_{+}\left((\Lambda y+f(y))^{-1}\right)\right) \\
& -g\left((\Lambda y+f(y))^{-1}\right)+\gamma_{+}\left((\Lambda y+f(y))^{-1}\right) .
\end{aligned}
$$

The second addend, $h_{-}$shell satisfy the equation

$$
\begin{aligned}
h_{-}(y) & =\Lambda^{-1} h_{-}(\Lambda y+f(y))+\Lambda^{-1} g\left(y+h_{-}(y)+h_{+}(y)\right) \\
& -\Lambda^{-1} g\left(y+h_{+}(y)\right)-\Lambda^{-1} \gamma_{-}(y),
\end{aligned}
$$

or, if we put $\tilde{g}(y)=\Lambda^{-1} g\left(y+h_{+}(y)\right)$,

$$
\begin{aligned}
h_{-}(y) & =\Lambda^{-1} h_{-}(\Lambda y+f(y))+\tilde{g}\left(y+h_{-}(y)\right) \\
& -\tilde{g}(y)-\Lambda^{-1} \gamma_{-}(y) .
\end{aligned}
$$

The existence of solutions to these equations follows from Lemma 6

It is known that one can define a "hat" function on a Hilbert space. It can be done in the following way: $\tau(y):=\tilde{\tau}\left(\|y\|^{2}\right)$, where $\tilde{\tau}(t)$ is a "hat" function in $\mathbb{R}$. Moreover, there exist maps $K_{ \pm}, K_{0}$, which can be defined as $K_{ \pm}:=\left(y_{ \pm} \cdot \tau\left(y_{ \pm}\right)\right)$, $K_{0}:=\left(y_{0} \cdot \tau\left(y_{0}\right)\right)$ and there exists a map $K:=\left(K_{+}, K_{-}, K_{0}\right)$. Then, the following corollary follows immediately from Theorem 1 .

Corollary 2. Assume that $B$ is a Hilbert space. Let

$$
F(y)=\Lambda y+f(y)
$$

and

$$
G(y)=\Lambda y+g(y)
$$

be two $C^{\infty}(B)$ diffeomorphisms. Assume that there exist $C^{\infty}$ non-expanding and non-contracting invariant manifolds. Also, assume that $f(y)=o(\|y\|), g(y)=$ $o(\|y\|)$ and $f(y)-g(y)=o\left(\left(\operatorname{dist}\left(y, L_{0}\right)\right)^{n}\right)$ for all $n \in \mathbb{N}$, as $\operatorname{dist}\left(y, L_{0}\right) \rightarrow 0$.

Then, there exists a $C^{\infty}(B)$ diffeomorphism $H(y)=y+h(y)$ with $h(y)=o(\|y\|)$, $h(y)=o\left(\left(\operatorname{dist}\left(y, L_{0}\right)\right)^{n}\right)$ for all $n \in \mathbb{N}$, as $\operatorname{dist}\left(y, L_{0}\right) \rightarrow 0$, such that for small values of $\|y\|$

$$
H \circ F=G \circ H \text {. }
$$

Remark 3. Note, that there exists $K$ on $C([0,1])$. It can be defined as $K(x)(t)=$ $\tau(x(t)) \cdot x(t)$. But it is unclear whether there exist $K_{ \pm}, K_{0}$ on the subspaces.

Remark 4. In fact, our proofs require the existence of only two $K$ maps: one $K$ map, defined on the entire space $B$ (for an extension of a diffeomorphism) and another $K$ map, defined on one of the subspaces $L_{+}$or $L_{-}$(for positive and negative splitting of some map, flat on $L_{0}$ ). If a diffeomorphism does not have either a contracting or an expanding part, then, we do not need to split the flat map into the positive and negative parts. 
Remark 5. Let $B=C([0,1])$. Consider purely non-contracting (or non-expanding) diffeomorphisms on $B$. Then, if the two diffeomorphisms formally conjugate, they smoothly conjugate in the class $C^{\infty}(B)$.

The same conclusion is true if at least one of the above mentioned subspaces is finite.

\section{SOlVABility in FLAT MAPS}

The following lemma is presented for the "non-positive" subspaces $L_{-, 0}, \mathcal{E}_{L_{-, 0}}$, etc. Proof for the "non-negative" subspaces is similar.

Lemma 6. Consider the equation, defined on a Banach space B,

$$
\begin{aligned}
\phi(y) & =A \phi(\Lambda y+f(y)) \\
& +g(\Lambda y+f(y)+\phi((\Lambda y+f(y))))-g(\Lambda y+f(y))+\rho(y)
\end{aligned}
$$

with a linear operator $A$, invertible linear operator $\Lambda$, a map $f \in C^{\infty}(B), f(y)=$ $o(\|y\|)$, as $\|y\| \rightarrow 0$ and a map $\rho$, which is flat on the linear non-expanding invariant manifold $L_{-, 0} \subset B$ of $\Lambda$. Then, there exists flat on $L_{-, 0}$ solution $\phi \in C^{\infty}(B)$.

Proof. We shell search for a fixed point of the operator $T$, defined by the right hand side of the equation 9 :

$$
\begin{aligned}
T_{\phi} & =A \phi(\Lambda y+f(y)) \\
& +g(\Lambda y+f(y)+\phi((\Lambda y+f(y))))-g(\Lambda y+f(y))+\rho(y)
\end{aligned}
$$

Consider space $\mathcal{E}_{L_{-, 0}}$ of smooth maps, flat on the manifold $L_{-, 0}$. To simplify our notations, we shell call it $\mathcal{E}$

Define a norm

$$
N_{k, p}(\phi):=\max _{0 \leq j \leq k} \sup \left\{\frac{\left\|\phi^{(j)}(y)\right\|}{\|\xi\|^{p}}, \phi \in \mathcal{E}, \xi \in L_{-}, y \in B\right\} .
$$

Proposition 7. There exist a real number $q<1$ and sequences of $b_{k, p} \in \mathbb{R}_{+}$and $p_{k} \in \mathbb{N}$, such that for all $p, k \in \mathbb{N}, p>p_{k}$ and $\phi, \psi \in \mathcal{E}$

1. $N_{0, p}\left(T_{\phi}\right) \leq q N_{0, p}(\phi)+N_{0, p}(\rho)$,

$N_{k, p}\left(T_{\phi}\right) \leq q N_{k, p}(\phi)+b_{k, p} N_{k-1, p}(\phi)+N_{k, p}(\rho)$ for $k=1,2, \ldots$ and

2. $\quad N_{0, p}\left(T_{\phi}-T_{\psi}\right) \leq q N_{0, p}(\phi-\psi)$,

$$
N_{k, p}\left(T_{\phi}-T_{\psi}\right) \leq q N_{k, p}(\phi-\psi)+b_{k, p} N_{k-1, p}(\phi-\psi) \text { for } k=1,2, \ldots
$$

Proof. First, we shell do the estimates for the part 2. The proof will require inductive arguments on the index $k$. It is easy to see that for $k=0$

$$
\begin{aligned}
N_{0, p}\left(T_{\phi}-T_{\psi}\right) & \leq\|A\| N_{0, p}(\phi(\Lambda y+f(y))-\psi(\Lambda y+f(y))) \\
& +\int_{0}^{1} g_{\sigma(s)}^{\prime} d s \cdot N_{0, p}(\phi(\Lambda y+f(y))-\psi(\Lambda y+f(y))) .
\end{aligned}
$$

Here $\sigma:[0,1] \rightarrow B$ denotes a path, connecting the points

$$
\sigma(0)=(\Lambda y+f(y)+\phi((\Lambda y+f(y))
$$

and

$$
\sigma(1)=(\Lambda y+f(y)+\psi((\Lambda y+f(y))))
$$


Then,

$$
N_{0, p}\left(T_{\phi}\right) \leq\left(\|A\|+\sup _{y}\left\|g^{\prime}(y)\right\|\right) \cdot N_{0, p}(\phi(\Lambda y+f(y))-\psi(\Lambda y+f(y))) .
$$

Let us recall that choosing sufficiently small $\delta$ in the $K$ function, we can make $\sup _{y}\left\|g^{\prime}(y)\right\|$ less than an arbitrary $\Delta$. (See formula 3.) Then,

$$
\begin{gathered}
N_{0, p}\left(T_{\phi}-T_{\psi}\right) \leq(\|A\|+\Delta) \cdot N_{0, p}(\phi(y)-\psi(y)) \\
\cdot\left(\sup _{y, \xi} \frac{\left\|(\Lambda y+f(y))_{\mid L_{-}}\right\|}{\|\xi\|}\right)^{p} .
\end{gathered}
$$

Since the spectrum of $\Lambda_{\mid L_{-}}$is less than 1 , and with sufficiently small $\delta$ in the $K$ function we can make $\|f(y)\|$ as small as we want (see formula 2), there exists $\alpha<1$ such that

$$
\sup _{y, \xi} \frac{\left\|(\Lambda y+f(y))_{\mid L_{-}}\right\|}{\|\xi\|}<\alpha<1 .
$$

Then, there exists $p_{0}$, such that for all $p>p_{0}$

$$
(\|A\|+\Delta) \cdot\left(\sup _{y, \xi} \frac{\left\|(\Lambda y+f(y))_{\mid L_{-}}\right\|}{\|\xi\|}\right)^{p}<q<<1 .
$$

Thus,

$$
N_{0, p}\left(T_{\phi}-T_{\psi}\right) \leq q N_{0, p}(\phi-\psi) .
$$

The estimates for $k=1$ are more complex.

$$
\begin{aligned}
N_{1, p}\left(T_{\phi}-T_{\psi}\right) \leq & \left(\|A\| \cdot N_{1, p}(\phi(y)-\psi(y)) \cdot\left\|(\Lambda y+f(y))^{\prime}\right\|\right. \\
& +\sup _{y}\left\|g^{\prime}\right\| N_{1, p}(\phi(y)-\psi(y)) \\
& \left.+\sup _{y}\left\|g^{\prime \prime}\right\| N_{0, p}(\phi(y)-\psi(y))\right) \\
& \cdot\left(\sup _{y, \xi} \frac{\left\|(\Lambda y+f(y))_{\mid L_{-}}\right\|}{\|\xi\|}\right)^{p} \\
& \leq\left(\|A\| \cdot\left\|(\Lambda y+f(y))^{\prime}\right\|+\epsilon\right) N_{1, p}(\phi(y)-\psi(y)) \\
& \cdot\left(\sup _{y, \xi} \frac{\left\|(\Lambda y+f(y))_{\mid L_{-}}\right\|}{\|\xi\|}\right)^{p}+b_{1, p} N_{0, p}(\phi(y)-\psi(y)) .
\end{aligned}
$$

Here the term $b_{1, p} N_{0, p}\left(\phi(y)-\psi(y)\right.$ ) (with some positive constant $b_{1, p}$ ) appears in the estimate, as a result of differentiation of the product of $\int_{0}^{1} g^{\prime} d s \cdot F(y)$. There exists $p_{1}$, such that for all $p>p_{1}$

$$
\left(\|A\| \cdot\left|(\Lambda y+f(y))^{\prime}\right|+\epsilon\right) \cdot\left(\sup _{y, \xi} \frac{\left\|(\Lambda y+f(y))_{\mid L_{-}}\right\|}{\|\xi\|}\right)^{p}<q<1 .
$$

Then, it follows that

$$
N_{1, p}\left(T_{\phi}-T_{\psi}\right)<q N_{1, p}(\phi-\psi)+b_{1, p} N_{0, p}(\phi-\psi) .
$$


THEOREM OF STERNBERG-CHEN MODULO CENTRAL MANIFOLD FOR BANACH SPACES

Suppose that the estimates are correct for $i=1, \ldots, k-1$. Then, for $i=k$

$$
\begin{aligned}
N_{k, p}\left(T_{\phi}-T_{\psi}\right) & \leq\left(\left(\|A\|+\left\|g^{\prime}\right\|\right) \cdot N_{k, p}(\phi(y)-\psi(y)) \cdot\left\|(\Lambda y+f(y))^{\prime}\right\|^{k}\right. \\
& \left.+b_{k, p} N_{k-1, p}(\phi(y)-\psi(y))\right) \\
& \cdot\left(\sup _{y, \xi} \frac{\left\|(\Lambda y+f(y))_{\mid L_{-}}\right\|}{\|\xi\|}\right)^{p} .
\end{aligned}
$$

Here the term $b_{k, p} N_{k-1, p}\left(\phi(y)-\psi(y)\right.$ ) (with some positive constant $b_{k, p}$ ) is related to taking the $k^{t h}$ derivative of the composition $(\phi-\psi) \circ F$, and estimating all addends with $(\phi-\psi)^{\prime}, \ldots,(\phi-\psi)^{(k-1)}$, plus the sum of the derivatives:

$$
\sum_{j=0}^{k-1} \frac{k !}{j !(k-j) !}\left\|g^{(j+1)}\right\| \cdot \sup _{x, \xi} \frac{\left\|(\phi-\psi)^{(k-j)}(y)\right\|}{\|\xi\|^{p}} .
$$

There exists $p_{k}$, such that for all $p>p_{k}$

$$
\left(\|A\|+\left\|g^{\prime}\right\|\right) \cdot\left|(\Lambda y+f(y))^{\prime}\right|^{k} \cdot\left(\sup _{y, \xi} \frac{\left\|(\Lambda y+f(y))_{\mid L_{-}}\right\|}{\|\xi\|}\right)^{p}<q<1 .
$$

Thus,

$$
N_{k, p}\left(T_{\phi}-T_{\psi}\right)<q N_{k, p}(\phi-\psi)+b_{k, p} N_{k-1, p}(\phi-\psi) .
$$

Thus, we proved part 2 .

Part 1 follows easily from part 2. Indeed,

$$
\begin{aligned}
N_{0, p}\left(T_{\phi}\right) & =N_{0, p}\left(T_{\phi}-T_{0}+\rho\right) \leq N_{0, p}\left(T_{\phi}-T_{0}\right)+N_{0, p}(\rho) \\
& <q N_{0, p}(\phi)+N_{0, p}(\rho) .
\end{aligned}
$$

and for $k=1,2, \ldots$

$$
\begin{aligned}
N_{k, p}\left(T_{\phi}\right) & =N_{k, p}\left(T_{\phi}-T_{0}+\rho\right) \leq N_{k, p}\left(T_{\phi}-T_{0}\right)+N_{k, p}(\rho) \\
& <q N_{k, p}(\phi)+b_{k, p} N_{k-1, p}(\phi)+N_{k, p}(\rho) .
\end{aligned}
$$

Using the above estimates, we introduce a new set of norms $\|.\|_{k, p}$, defined with the help of $N_{k, p}$, such that the operator $T$ is a contraction in $\|.\|_{k, p}$.

Let $\|.\|_{k, p}$ be defined on $\mathcal{E}$ according to the following rule:

$$
\begin{aligned}
& \|\phi\|_{0, p}:=N_{0, p}(\phi) \\
& \|\phi\|_{1, p}:=N_{1, p}(\phi)+A_{1, p}^{1} N_{0, p}(\phi) \\
& \vdots \\
& \|\phi\|_{k, p}:=N_{k, p}(\phi)+A_{k, p}^{1} N_{k-1, p}(\phi)+A_{k, p}^{2} N_{k-2, p}(\phi)+\ldots+A_{k, p}^{k} N_{0, p}(\phi) .
\end{aligned}
$$

Choosing appropriate constants $A_{k, p}^{j}$, one can show that the operator $T$ is a contraction in each norm $\|\cdot\|_{k, p}$.

Proposition 8. Let $q$ be as in Proposition 7. Fix $\epsilon>0$ such that $q+\epsilon<1$. Let the constants $A_{k, p}^{j}(j=1, \ldots, k)$ in the definition of the norms $\|\cdot\|_{k, p}$ satisfy the 
following inequalities:

$$
\begin{aligned}
& A_{k, p}^{1}>\frac{b_{k, p}}{\epsilon} \\
& A_{k, p}^{2}>\frac{A_{k, p}^{1} b_{k-1, p}}{\epsilon} \\
& \vdots \\
& A_{k, p}^{k}>\frac{A_{k, p}^{k-1} b_{1, p}}{\epsilon}
\end{aligned}
$$

Then, for each pair of $\phi, \psi \in \mathcal{E}$ and for each norm $\|\cdot\|_{k, p}\left(p>p_{k}\right)$, the operator $T$ admits the following estimates:

$1^{\prime} . \quad\left\|T_{\phi}\right\|_{k, p}<(q+\epsilon)\|\phi\|_{k, p}+\|\rho\|_{k, p}$ and

$2^{\prime}$. $\left\|T_{\phi}-T_{\psi}\right\|_{k, p}<(q+\epsilon)\|\phi-\psi\|_{k, p}$.

Proof. We begin with the second part of the proposition. In this proof we shell also use induction on the $k$-index.

$$
\left\|T_{\phi}-T_{\psi}\right\|_{0, p}=N_{0, p}\left(T_{\phi}-T_{\psi}\right)<q N_{0, p}(\phi-\psi)=q\|\phi-\psi\|_{0, p} .
$$

Thus, it is a contraction in $\|\cdot\|_{0, p}$.

$$
\begin{aligned}
\left\|T_{\phi}-T_{\psi}\right\|_{1, p} & =N_{1, p}\left(T_{\phi}-T_{\psi}\right)+A_{1, p}^{1} N_{0, p}\left(T_{\phi}-T_{\psi}\right) \\
& <q N_{1, p}(\phi-\psi)+b_{1, p} N_{0, p}(\phi-\psi)+A_{1, p}^{1} q N_{0, p}(\phi-\psi) \\
& =q N_{1, p}(\phi-\psi)+\left(A_{1, p}^{1} q+b_{1, p}\right) N_{0, p}(\phi-\psi) \\
& <(q+\epsilon) N_{1, p}(\phi-\psi)+A_{1, p}^{1}(q+\epsilon) N_{0, p}(\phi-\psi),
\end{aligned}
$$

if $A_{1, p}^{1}>\frac{b_{1, p}}{\epsilon}$. Finally, note that

$$
(q+\epsilon) N_{1, p}(\phi-\psi)+A_{1, p}^{1}(q+\epsilon) N_{0, p}(\phi-\psi)=(q+\epsilon)\|\phi-\psi\|_{1, p} .
$$

Thus, in this norm, the operator $T$ is a contraction as well.

Assume that the assertion of the Proposition is correct for all $i=1, \ldots, k-1$. We 
THEOREM OF STERNBERG-CHEN MODULO CENTRAL MANIFOLD FOR BANACH SPACE 9 shell prove it for $i=k$, i.e., we shell estimate $\left\|T_{\phi}-T_{\psi}\right\|_{k, p}$.

$$
\begin{aligned}
\left\|T_{\phi}-T_{\psi}\right\|_{k, p} & =N_{k, p}\left(T_{\phi}-T_{\psi}\right)+A_{k, p}^{1} N_{k-1, p}\left(T_{\phi}-T_{\psi}\right) \\
& +A_{k, p}^{2} N_{k-2, p}(T \phi-T \psi)+\ldots+A_{k, p}^{k} N_{0, p}(T \phi-T \psi) \\
& <q N_{k, p}(\phi-\psi)+b_{k, p} N_{k-1, p}(\phi-\psi) \\
& +A_{k, p}^{1}\left[q N_{k-1, p}(\phi-\psi)+b_{k-1, p} N_{k-2, p}(\phi-\psi)\right] \\
& +A_{k, p}^{2}\left[q N_{k-2, p}(\phi-\psi)+b_{k-2, p} N_{k-3, p}(\phi-\psi)\right] \\
& \vdots \\
& +A_{k, p}^{k-1}\left[q N_{1, p}(\phi-\psi)+b_{1, p} N_{0, p}(\phi-\psi)\right] \\
& +A_{k, p}^{k} q N_{0, p}(\phi-\psi) \\
& =q N_{k, p}(\phi-\psi) \\
& +\left(A_{k, p}^{1} q+b_{k, p}\right) N_{k-1, p}(\phi-\psi) \\
& +\left(A_{k, p}^{2} q+A_{k, p}^{1} b_{k-1, p}\right) N_{k-2, p}(\phi-\psi) \\
& \vdots \\
& +\left(A_{k, p}^{k-1} q+A_{k, p}^{k-2} b_{2, p}\right) N_{1, p}(\phi-\psi) \\
& +\left(A_{k, p}^{k} q+A_{k, p}^{k-1} b_{1, p}\right) N_{0, p}(\phi-\psi)
\end{aligned}
$$

From inequalities 11 it immediately follows that

$$
\left(A_{k, p}^{1} q+b_{k, p}\right)<(q+\epsilon) A_{k, p}^{1}
$$

and

for $j=0, \ldots, k-2$. Then,

$$
A_{k, p}^{k-j} q+A_{k, p}^{k-j-1} b_{j+1, p}<(q+\epsilon) A_{k, p}^{k-j}
$$

$$
\begin{aligned}
\left\|T_{\phi}-T_{\psi}\right\|_{k, p} & <q N_{k, p}(\phi-\psi) \\
& +A_{k, p}^{1}(q+\epsilon) N_{k-1, p}(\phi-\psi) \\
& +A_{k, p}^{2}(q+\epsilon) N_{k-2, p}(\phi-\psi) \\
& \vdots \\
& +A_{k, p}^{k-1}(q+\epsilon) N_{1, p}(\phi-\psi) \\
& +A_{k, p}^{k}(q+\epsilon) N_{0, p}(\phi-\psi), \\
& <(q+\epsilon)\left[N_{k, p}+A_{k, p}^{1} N_{k-1, p}+\ldots+A_{k, p}^{k} N_{0, p}\right] \\
& =(q+\epsilon)\|\phi-\psi\|_{k, p}
\end{aligned}
$$

Thus the first part of the theorem is proved. The second part follows immediately from the first part. Indeed,

$$
\left\|T_{\phi}\right\|_{k, p}=\left.\left\|T_{\phi}-\left(T_{0}-\rho\right)\right\|\right|_{k, p} \leq\left\|T_{\phi}-T_{0}\right\|_{k, p}+\|\rho\|_{k, p} \leq(q+\epsilon)\|\phi\|\left\|_{k, p}+\right\| \rho \|_{k, p} .
$$

Now, we shell define a closed disk, such that it is invariant under the action of $T$. Choose a sequence of real positive numbers $c_{k, p}$, and define the closed disc $D$ in the space $\mathcal{E}$ as

$$
D=\left\{\phi \in \mathcal{E}:\|\phi\|_{k, p} \leq c_{k, p}\right\} .
$$


Assume that for some small $\epsilon>0$, the constants $c_{k, p}$ are such that

$$
c_{k, p} \geq \frac{\|\rho\|_{k, p}}{1-q-\epsilon} \text {. }
$$

\section{Corollary 9.}

$$
T(D) \subset D
$$

Proof. The proof is an immediate consequence of the estimate $1^{\prime}$ in Proposition 8.

Thus, we have proved that in the countable collection of norms $\|\cdot\|_{k, p}$, the operator $T$ is a contraction that maps the closed disc $D$ into itself. Using these inequalities, we can now construct a metric on $\mathcal{E}$ with the properties, similar to those, discussed for the norms $\|.\|_{k, p}$. Then, we can use Banach Contraction Principle to show the existence of a fixed point of the operator $T$ in the $\operatorname{disc} D$.

Let us define the metric on $D$. Fix a positive $R \in \mathbb{R}$. Let $\epsilon_{k, p}<1$ be a sequence of positive real numbers, such that $c_{k, p} \epsilon_{k, p}<R$ (here $c_{k, p}$ are the constants that define the $\operatorname{disc} D)$. Then,

$$
\rho(\phi, \psi):=\sum_{k, p>p_{k}} \frac{1}{2^{k p}} \cdot \frac{\epsilon_{k, p}\|\phi-\psi\|_{k, p}}{1+\epsilon_{k, p}\|\phi-\psi\|_{k, p}}, \quad \phi, \psi \in D .
$$

Obviously, $D$ is a complete metric space.

Consider a function $\kappa(t)=\frac{t}{1+t}$, defined for positive values of $t$. This is an increasing function, it is less than 1 for bounded values of $t$. Put

$$
t:=\epsilon_{k, p}\|\phi-\psi\|_{k, p}<R .
$$

Since $T$ is a contraction in $\|\cdot\|_{k, p}$, and $\kappa(t)$ is an increasing function,

$$
\rho\left(T_{\phi}, T_{\psi}\right)<\sum_{k, p>p_{k}} \frac{1}{2^{k p}} \cdot \frac{(q+\epsilon) \epsilon_{k, p}\|\phi-\psi\|_{k, p}}{1+(q+\epsilon) \epsilon_{k, p}\|\phi-\psi\|_{k, p}} .
$$

Also for bounded values of $t$ there exists $0<r<1$ such that

$$
\kappa((q+\epsilon) t)<r \kappa(t) .
$$

In other words,

$$
\frac{(q+\epsilon) \epsilon_{k, p}\|\phi-\psi\|_{k, p}}{1+(q+\epsilon) \epsilon_{k, p}\|\phi-\psi\|_{k, p}}<r \frac{\epsilon_{k, p}\|\phi-\psi\|_{k, p}}{1+\epsilon_{k, p}\|\phi-\psi\|_{k, p}} .
$$

We proved that $T: D \rightarrow D$ acts as a contraction operator on $D$ :

$$
\rho\left(T_{\phi}, T_{\psi}\right)<r \rho(\phi, \psi)
$$

The Banach Contraction Principle implies the existence of a fixed point in $D$. This fixed point is the solution of the equation 10 .

In the proof of Theorem 1 we used the fact that any map, which is flat on the central manifold can be represented as a sum of two maps, flat on the linear subspaces. In the next lemma we discuss how to split $\gamma$ into the sum of $\gamma_{+}$and $\gamma_{-}$.

Lemma 10. Let $\gamma(y) \in C^{\infty}(B)$ be flat on $L_{0}$. Let $L_{-}$and $L_{+}$be contracting and expanding subspaces of $B$. Suppose that there exists a $C^{\infty} K$ map on one of the subspaces. Then, there exist maps $\gamma_{+}$and $\gamma_{-}$, such that $\gamma=\gamma_{-}+\gamma_{+}$, and $\gamma_{+}$is flat on $L_{-, 0}$ and $\gamma_{-}$is flat on $L_{+, 0}$. 
Proof. Lets write $y=(\xi, \eta, t) \in B$ with $\xi \in L_{+}, \eta \in L_{-}$and $t \in L_{0}$. For definiteness, lets assume that there exists $C^{\infty} K$ map on $L_{-, 0}$. Then, we can explicitly construct $\gamma_{+}$map.

Let $\delta_{j} \neq 0$ be so small that the following series converges in $C^{\infty}$ topology.

$$
\gamma_{+}:=\sum_{j=0}^{\infty} \frac{\partial^{j} \gamma}{\partial \eta^{j}}(\xi, 0, t)\left(K\left(\delta_{j}^{-1} \eta\right), \ldots, K\left(\delta_{j}^{-1} \eta\right)\right) \cdot \frac{\delta_{j}^{j}}{j !}
$$

Define $\gamma_{-}$as

$$
\gamma_{-}=\gamma-\gamma_{+}
$$

Then, it is easy to check that $\gamma_{+}$is flat on $L_{+, 0}$ and $\gamma_{-}$is flat on $L_{-, 0}$. Indeed,

$$
\begin{aligned}
\frac{\partial^{p} \gamma_{+}}{\partial \xi^{p}}(0, \eta, t) & =\sum_{j=0}^{\infty} \frac{\partial^{j} \gamma}{\partial \eta^{j}}(0,0, t)\left(K\left(\delta_{j}^{-1} \eta\right), \ldots, K\left(\delta_{j}^{-1} \eta\right)\right) \cdot \frac{\delta_{j}^{j}}{j !} \\
& =0\left(K\left(\delta_{j}^{-1} \eta\right), \ldots, K\left(\delta_{j}^{-1} \eta\right)\right) \cdot \frac{\delta_{j}^{j}}{j !}=0 .
\end{aligned}
$$

Also,

$$
\frac{\partial^{q} \gamma_{-}}{\partial \eta^{q}}(\xi, 0, t)=\frac{\partial^{q} \gamma}{\partial \eta^{q}}(\xi, 0, t)-\sum_{j=0}^{\infty} \frac{\partial^{q}}{\partial \eta^{q}}\left(\frac{\partial^{j} \gamma}{\partial \eta^{j}}(\xi, 0, t)\left(K\left(\delta_{j}^{-1} \eta\right), \ldots, K\left(\delta_{j}^{-1} \eta\right)\right)\right) \cdot \frac{\delta_{j}^{j}}{j !}
$$

Recall that $K^{(j)}(0)=\mathbf{0}$ for $j \neq 1$ and $K^{\prime}(0)=\mathbf{1}$. Then,

$$
\frac{\partial^{q} \gamma_{-}}{\partial \eta^{q}}(\xi, 0, t)=\frac{\partial^{q} \gamma}{\partial \eta^{q}}(\xi, 0, t)-\frac{\partial^{q} \gamma}{\partial \eta^{q}}(\xi, 0, t) \cdot \mathbf{1} \cdot \delta_{k}^{-k} \cdot \delta_{k}^{k}=0
$$

\section{LiNEARIZATION}

In this section we shell prove that a linearization is a $C^{\infty}$ map on a Banach space, if non-resonance condition is satisfied.

Now we shell formally define the resonance for diffeomorphisms on Banach spaces. Let $\Lambda_{1}$ be a hyperbolic linear operator on a Banach space $B_{1}$. Consider the following linear operator $L_{k}$, defined on the space of homogeneous polynomial maps of degree $k$.

$$
\begin{aligned}
& L_{k}: P^{(k)}\left[B_{1}\right] \rightarrow P^{(k)}\left[B_{1}\right], \\
& L_{k} \phi(x):=\Lambda_{1} \phi(x)-\phi\left(\Lambda_{1} x\right) .
\end{aligned}
$$

Definition 11. Suppose that for all $k \in \mathbb{N}, k>1, L_{k}$ is invertible on $P^{(k)}\left[B_{1}\right]$. Then, we say that the operator $\Lambda_{1}$ has no resonances.

In particular, when $B_{1}$ is a $n$-dimensional space, the non-resonance condition is equivalent to the following statement:

For any $\lambda_{j} \in \sigma\left(\Lambda_{1}\right)$ and for any combination of $p_{1}, \ldots, p_{n} \in \mathbb{N}, p_{1}+\ldots+p_{n}>1$

$$
\lambda_{j}-\lambda_{1}^{p_{1}} \ldots \lambda_{n}^{p_{n}} \neq 0
$$

Now we present $C^{\infty}$ linearization theorem on Banach spaces for a family of diffeomorphisms with a hyperbolic fixed point. The proof of this theorem easily follows from Theorem 1 . 
Theorem 12. Let

$$
F(y)=\Lambda y+f(y)=F_{t}(x)=\left\{\begin{array}{l}
\Lambda_{1} x+f_{t}(x) \\
t
\end{array}\right.
$$

be a $C^{\infty}\left(B_{1} \times B_{2}\right)$ family of diffeomorphism on the product of Banach spaces $B_{1}$ and $B_{2}$. Assume that $\Lambda_{1}$ is hyperbolic and $f_{t}(y)=o_{t}(\|y\|)$. Also, assume that there exist $K_{ \pm}$maps on the corresponding subspaces $L_{ \pm}$, and $\Lambda_{1}$ has no resonances.

Then, there exists a family of $C^{\infty}\left(B_{1} \times B_{2}\right)$ diffeomorphisms

$$
H(y)=y+h(y)=H_{t}(x)=\left\{\begin{array}{l}
x+h_{t}(x) \\
t
\end{array}\right.
$$

with $h_{t}(x)=o_{t}(x)$, as $\|x\| \rightarrow 0$, such that for small values of $\|x\|$ and $\|t\|$

$$
H_{t} \circ F_{t}=L H_{t} .
$$

Proof. Let $H(y)=y+h(y)$. Then, the equation 12 can be simplified:

$$
f(y)+h(\Lambda y+f(y))=\Lambda h(y)
$$

In order to get a flat residue, let us formally differentiate both sides of the hyperbolic part of this equation

$$
\left[f_{t}(x)+h_{t}\left(\Lambda_{1} x+f_{t}(x)\right)\right]_{x=0}^{(k)}=\Lambda_{1} h_{t}^{(k)}(x)_{x=0}
$$

and solve it for $h_{t}^{(k)}(0)$. The solution $h_{t}^{(k)}(0)$ exists, if $\Lambda_{1}$ has no resonances.

Now, with the help of the Borel lemma, we can construct a map $\tilde{h}_{t}(x)$ on the whole space with the same derivatives at 0 . Since there exist $K$ map on $B$, the Borel lemma is valid on the space $B$. The proof of the Borel lemma for Banach spaces can be found in [B3] (these ideas were also used in [B1]). Lets write $h_{t}(x)$ as the sum of $\tilde{h}_{t}(x)$ and a flat map $\phi_{t}(x)$. Then the equation 13 can be written as

$$
\Lambda_{1} \phi_{t}(x)-\phi_{t}\left(\Lambda_{1} x+f_{t}(x)\right)=f_{t}(x)+\tilde{h_{t}}\left(\Lambda x+f_{t}(x)\right)-\Lambda_{1} \tilde{h}_{t}(x) .
$$

The right hand side of this equation is flat at 0 . Then, by Lemma 6 we can solve it for $\phi_{t}$.

Let us say a few words about applications of the linearization theorems. How strict is the non-resonance condition? Obviously there are many examples of nonresonant maps, if the space $B$ has finite dimension. Here we shell construct a non-resonant discrete spectrum in infinite dimensions. Consider integers $\left\{p_{j}\right\}_{j=1}^{m}$ and $\left\{s_{j}\right\}_{j=1}^{m}$, which are all relatively prime. Let $r_{j}=\frac{p_{j}}{s_{j}}$. Define the spectrum of $\Lambda$ as

$$
\sigma(\Lambda)=\left\{\lambda_{t}=r_{j_{t}} e^{i \phi_{t}}, j_{t} \in\{1, \ldots, m\}, \phi_{t} \in \mathbb{T}\right\},
$$

and

$$
\Lambda=\operatorname{diag}\left(\lambda_{t}\right) .
$$

In this example, we may have infinitely many distinct eigenvalues, but their absolute values form a finite set of positive rational numbers $r_{j}$. Since the product of any combination of $r_{j}$ 's cannot be equal to one of the $r_{j}$, the linear operator $\Lambda$ has no resonances.

We can also construct a continuous non-resonant spectrum. Suppose the unit circle does not separate the spectrum, and $0<a<\left|\sigma\left(\Lambda_{1}\right)\right|<b<\infty$. If $a>1$, i.e., $\Lambda$ is an expansion, then it is enough to assume that $a^{2}>b$. Otherwise, if $b<1$, so that $\Lambda$ is a contraction, it is enough to assume that $b^{2}<a$. 
THEOREM OF STERNBERG-CHEN MODULO CENTRAL MANIFOLD FOR BANACH SPACE\$

Similar, one can construct an example with a contracting (expanding) discrete non-resonant spectrum.

\section{REFERENCES}

[B] G. Belitskii: Functional equations, and local conjugacy of mappings of class $C^{\infty}$. Math USSR-Sb. 20 (1973), 587-602.

[B1] G. Belitskii: The Sternberg theorem for a Banach space. Functional Anal. Appl. 18 (1984) no. 3, 238-239.

[B2] G. Belitskii: The equivalence of families of local diffeomorphisms. Functional Anal. Appl. 8 (1974) no. 4, 338-339.

[B3] G. Belitskii: Borel's theorem in a Banach space, (Russian) Mathematical physics, functional analysis (Russian), 148, "Naukova Dumka", Kiev, (1986) 137-139

[B4] G. Belitskii: Normal forms, invariant and local mappings, (Russian) "Naukova Dumka", Kiev, 1979. $173 \mathrm{pp}$

[Br] A. Brjuno: Analytic form of differential equations Transactions of the Moscow Mathematical Society for the year 1971 (Vol. 25).

[Ch] K. Chen: Equivalence and decomposition of vector fields about an elementary critical points. Amer. J. of Math. 85 no. 4, 693-722. Bull. Soc. Math. France 40 (1912), 324-383

[H] P. Hartman: On local homeomorphisms of Euclidean spaces, Bol. Soc. Mat. Mexicana (2) 5 (1960) 220-241

[N] Z. Nitecki: Differentiable dynamics. An introduction to the orbit structure of diffeomorphisms. The M.I.T. Press, Cambridge, Mass.-London, 1971.

[R] V. Rayskin: Smooth dependence of linearization on perturbations. Mathematical Physics Preprint Archive, mp_arc 06-92 (2006).

[RS-M] H. Rodrigues and J. Solà-Morales: Invertible Contractions and Asymptotically Stable $O D E$ 'S that are not $C^{1}$-Linearizable Journal of Dynamics and Differential Equations (2006)

[RS-M1] H. Rodrigues and J. Solà-Morales: Linearization of calss $C^{1}$ for contractions on Banach spaces. J. Diff. Equat. 201(2), 351-382.

[S] S. Sternberg: Local contractions and a theorem of Poincaré, Amer. J. of Math. 79 (1957) no. 4

Department of Mathematics, Ben-Gurion University of the Negev, P.O.B. 653, BeerSheva 84105, IsRael

E-mail address: vrayskin@gmail.com 